Carnets de géographes

GÉOGRAPHES

\title{
L'économie de la vitesse : l'automobilisme et ses enjeux dans le département du Rhône et la région de Québec (1919-1961)
}

Etienne Faugier

\section{CpenEdition}

Journals

Édition électronique

URL : http://journals.openedition.org/cdg/898

DOI : $10.4000 /$ cdg. 898

ISSN : 2107-7266

Éditeur

UMR 245 - CESSMA

Référence électronique

Etienne Faugier, « L'économie de la vitesse : l'automobilisme et ses enjeux dans le département du Rhône et la région de Québec (1919-1961) », Carnets de géographes [En ligne], 6 | 2013, mis en ligne le 17 janvier 2017, consulté le 24 septembre 2020. URL : http://journals.openedition.org/cdg/898 ; DOI : https://doi.org/10.4000/cdg.898

\section{(c) (†) $\odot$}

La revue Carnets de géographes est mise à disposition selon les termes de la Licence Creative Commons Attribution - Pas d'Utilisation Commerciale - Pas de Modification 4.0 International. 


\section{L'économie de la vitesse : l'automobilisme et ses enjeux dans le département du Rhône et la région de Québec (1919-1961)}

\section{ETIENNE FAUGIER}

Ce travail de doctorat en cotutelle s'attache à analyser les impacts de l'automobilisme - soit les véhicules à moteur et leurs usages - dans l'espace rural du département du Rhône et de la région de Québec au XX ${ }^{\mathrm{e}}$ siècle. L'objectif de notre travail consistait à mettre en lumière la conquête d'un socio-système tel que l'automobilisme dans un espace - l'espace rural - jusqu'à présent resté dans l'angle mort de la recherche en histoire des transports et de la mobilité.

Pour la pertinence de notre propos, nous sommes passés par diverses étapes méthodologiques, conceptuelles et documentaires. Tout d'abord, mettre en relation le département du Rhône et la région de Québec nous a conduits à évaluer les ressemblances, les dissemblances et les différences entre ces deux territoires. II s'avère qu'ils demeurent relativement similaires quant à la structuration géographique, l'agriculture, l'exode rural, l'économie pour permettre une mise en relation fertile. La région de Québec n'étant pas un territoire politiquement et administrativement défini, en utilisant l'historiographie, nous l'avons défini comme regroupant les comtés de Portneuf, de Montmorency, de l'île d'Orléans et la ville de Québec. Pour le cas du département du Rhône, nous avons sélectionné l'espace administratif défini par le politique durant le $\mathrm{XX}$ e siècle.

Pour éclaircir notre problématique, nous avons sollicité diverses sources. Tout d'abord les sources issues des archives gouvernementales témoignant des travaux publics et de la politique automobile. Puis, les périodiques spécialisés, les revues des clubs automobiles, la presse de l'époque, l'iconographie, les guides touristiques ont été parcourus. Collectés au cours de divers voyages transatlantiques, ils ont été analysés un à un, puis confrontés et croisés au sein de chacun des territoires et au regard de l'autre terrain d'étude.

Afin de mettre en relation les deux territoires prédéfinis, nous avons utilisé la méthodologie de l'histoire croisée. Pour ce faire, nous avons construit une définition du concept de vitesse que nous avons surimposé aux deux espaces. La vitesse, selon nous, se fonde nécessairement autour d'un quatuor logique et cohérent composé d'un mobile - soit l'automobile et les autres véhicules à moteur -, d'une infrastructure, d'une normalisation et de pratiques socioculturelles. Il s'agit donc d'un système logique et cohérent. 
Notre démonstration présente quatre temps. En premier, nous proposons de contextualiser la vitesse en croisant les deux territoires pour la période couvrant la fin du XIXe siècle et la fin de la Première Guerre mondiale. La seconde partie s'attache à analyser l'évolution de l'infrastructure routière et ses abords en mettant en valeur la naissance et la construction d'une politique automobile. L'aménagement du territoire s'engage à la lumière de l'automobilisme au travers de la signalisation, de la faune, des infrastructures économiques. Par la suite, nous mettons en évidence l'essor de la culture automobile qui rend possible l'automobilité : il s'agit à la fois d'acquérir des connaissances concernant le véhicule, son utilisation, l'espace environnant, mais aussi la normalisation qui a cours. Enfin, nous prêtons un œil attentif aux pratiques de mobilité issues de l'usage des véhicules à moteur que sont l'automobile, le poids lourd, l'autobus, le tracteur. II est aussi question des enjeux sociétaux et culturels que suscite l'automobilisme dans le champ du tourisme, de l'alimentation et de la religion.

Ce travail est agrémenté d'une quarantaine de cartes de la région de Québec et du département du Rhône traitant des infrastructures économiques - garages, pompes à essence, hôtels, circuits de la Poste Automobile Rurale - et des véhicules à moteur - nombre de tracteurs, de véhicules agricoles, de chevaux. II est aussi illustré par divers documents iconographiques, des tableaux statistiques et des documents textuels.

L'histoire croisée peut constituer une approche pertinente pour les géographes. Dans la lignée des travaux d'Emmanuel Perrin sur l'automobile à Lyon, Lille et Stuttgart (2004), la méthodologie que nous avons choisie peut être reproductible pour les travaux qui souhaitent embrasser une approche globale. Par ailleurs, le concept de vitesse tel que défini peut être réutilisé pour souligner la problématique de l'aménagement du territoire.

Le croisement du département du Rhône et de la région de Québec s'avère fertile d'enseignements. Les mutations imposées par l'automobilisme sont, quoique moins visibles parfois, bien plus nombreuses et complexes au sein du monde rural qu'elles peuvent l'être dans l'espace urbain. II présente également la rapidité avec laquelle les populations rurales ont adopté ce mode de transport : la question de l'anti-automobilisme rural est largement repensée et une attention est portée aux impacts socioculturels de la mécanisation agricole. La vitesse issue de l'automobilisme uniformise les deux territoires en les insérant dans la même trajectoire et une chronologie qui se recoupe après 1945 . On observe une homogénéisation de l'offre et de la demande automobile, des mutations environnementales, politiques et culturelles au sein des deux sociétés étudiées. 


\section{Discipline}

Histoire

\section{Directeur}

Claude-Isabelle Brelot, Université Lumière Lyon 2

Martin Pâquet, Université Laval, Québec

\section{Université}

Université Lumière Lyon 2

Université Laval, Québec

Membres du jury de thèse, soutenue le 22 mars 2013

- Jean-Luc Mayaud, Université Lumière Lyon 2

- Claude-Isabelle Brelot, Université Lumière Lyon 2

- Martin Pâquet, Université Laval

- Marc Vallières, Université Laval

- Laurent Tissot, Université Neuchâtel

\section{Situation professionnelle}

Post-doctorant, Université de Neuchâtel

\section{Courriel de l'auteur}

etienne.faugier@gmail.com 